rev.relac.int.estrateg.segur.6(1):89-109,2011

\title{
LA GOBERNANZA VERSUS GLOBALIZACIÓN: ESTUDIO DE CASO ISA
}

\author{
Carlos Manuel Jiménez Aguilar* \\ Catherine Pereira Villa**
}

\section{RESUMEN}

Este trabajo explora el debate contemporáneo sobre globalización y gobernanza, con el objeto de estudiar procesos de integración regional a partir de las redes de gobernanza transfronteriza en la región. Los procesos de privatización del sector eléctrico en América Latina aumentaron la coordinación público-privada y las alianzas intersectoriales: este es el caso del sector energético colombiano y de la empresa de Interconexión Eléctrica ISA. Este trabajo sugiere que el caso de ISA se puede caracterizar como uno de gobernanza interactiva que ha interconectado tres países de la región andina y uno del Mercosur a través de una infraestructura lineal de más de 35.000 km. Esta infraestructura ha integrado espacialmente una región caracterizada por procesos incompletos de integración. El estudio aborda el modelo de la compañía y su contribución a la gobernanza transfronteriza a partir de información aportada por la empresa y una serie de entrevistas con varios directivos.

* Profesor de la Escuela Internacional de Ciencias Económicas y Administrativas de La Universidad de la Sabana.carlosja@unisabana.edu.co

** Jefe del Área de Negociación y Comercio Internacional. Escuela Internacional de Ciencias Económicas y Administrativa Universidad de la Sabana. catherine.pereira@unisabana.edu.co 
Palabras clave: gobernanza transfronteriza, interconexión eléctrica, integración regional, coordinación público-privada, alianzas intersectoriales.

\section{ABSTRACT}

This article explores the contemporary debate on globalization and governance, in order to study processes of regional integration from networks of cross-border governance in the region. The processes of privatization of the electric sector in Latin America increased public-private coordination and cross-sector partnerships: this is the case of Colombia's energy sector and the company of electrical interconnection (ISA). This work suggests that the case of ISA can be characterized as one of interactive governance that has connected three countries of the Andean region and one member of Mercosur through a linear infrastructure of more than 35,000 km. This infrastructure has spatially integrated a region characterized by incomplete integration processes. This study analyses ISA's model and its contribution to the cross-border governance based on information provided by the company and a series of interviews with ISA's management.

Keywords: cross-border governance, electrical interconnection, regional integration, public-private coordination, cross-sector alliances.

\section{RESUMO}

Este artigo explora o debate contemporâneo sobre a globalização e a governança, com o objetivo de estudar os processos de integração regional a partir das redes de governaças transfronteriças na região. Os processos de privatização do setor elétrico na América Latina aumentaram a coordenação público-privada e as parcerias intersetoriais: este é o caso do setor energético colombiano e da empresa de Interconexión Eléctrica (ISA). O presente artigo sugere que o caso da ISA pode se caracterizar como uma governança interativa que conectou três países da região andina e um do Mercosul através de uma infraestrutura linear superior a $35.000 \mathrm{~km}$. Esta infraestrutura integrou espacialmente uma região caracterizada por processos de integração incompletos. O estudo aborda o modelo da companhia e sua contribuição à governança transfronteriça com base na informação fornecida pela empresa e uma série de entrevistas com vários executivos.

Palavras chave: governança transfronteriça, interconexão elétrica, integração regional, coordenação público-privada, parcerias intersetoriais. 


\section{INTRODUCCIÓN}

Este documento explora el concepto de gobernanza transfronteriza como una herramienta conceptual para repensar los procesos regionales de inserción en la globalización, a través del caso empresarial de gobernanza de redes de interconexión de infraestructura lineal de la empresa colombiana Interconexión Eléctrica ISA. Por gobernanza transfronteriza se entiende en un sentido amplio la coordinación publico-privada y la regulación de un sistema societal. Bajo el modelo de interconexión espacial que ISA opera en Colombia, Brasil, Perú, Ecuador y Bolivia, se pretende abrir la discusión sobre el potencial de la gobernanza transfronteriza en la región y describir su proceso de interconexión, estrategias y mecanismos de integración en un subcontinente en busca de la consolidación de su bienestar y competitividad.

Las estrategias empresariales de ISA frente a la gobernanza en la región muestran las posibilidades que tiene el sector privado, en coordinación con el sector público, en la gestión de mercados de servicios. La gobernanza transfronteriza como estrategia de gestión de interconexión implica un amplio rango de socios en distintos sectores y niveles de organización territorial, donde actores públicos y privados se configuran como socios y activadores (Kohler-Koch, 2004). De esta manera, el sector privado, los agentes públicos, la sociedad civil y la comunidad internacional participan como socios y árbitros de iniciativas agenciadas en red por el sector privado en alianza con los demás actores. En este sentido, la pregunta de este texto se formula en términos de ¿̇cuáles han sido las características del modelo de interconexión eléctrica que pueden contribuir a la gobernanza transfronteriza en la región?

La pregunta se aborda a partir del proceso de interconexión eléctrica regional que gestiona una red de infraestructura de transporte punto a punto por fuera de la jurisdicción territorial nacional. La exploración de una visión internacional de gobernanza plantea una reflexión sobre el potencial derivado de la toma de decisiones y la gestión coordinada entre el sector público y el privado como eje central de nuevos desafíos técnicos y administrativos de alcance regional y de nuevas y transformadoras representaciones del espacio, más allá de los límites de las fronteras nacionales como compartimentos "naturales" de lo político y lo económico. De esta manera, el tema plantea una discusión sobre lo que entendemos en la región por globalización e integración a través de nuevos modelos de gobernanza empresarial en un espacio regional.

Existen pocos estudios sobre la gobernanza transfronteriza en Colombia y este no ha sido lo suficientemente estudiado ni abordado a partir de estudios de caso que permitan ampliar la comprensión de las estrategias y el potencial económico y administrativo de la gobernanza regional. Por el contario, el tema ha sido tratado de manera indirecta, supeditado al análisis de los mecanismos binacionales y a la gestión de los marcos subregionales de integración provistos por la Comunidad Andina CAN (Ramírez, 2002; González \& Ramírez, 2006; Vieira, 2008).

La gobernanza ha sido analizada indirectamente a partir de las dinámicas económicas binacionales derivadas del intercambio económico y la penetración empresarial en mercados vecinos, con 
especial atención en el mercado venezolano por razones del nivel de transacciones y la importancia de Venezuela dentro de la antigua CAN (Cardozo \& Romero, 1999; García \& Urdaneta, 1999; Beltrán, 2006; Londoño, 2008; Mojica, 2004; Montes \& Garavito, 2008). Otros trabajos han estudiado el tema desde una perspectiva financiera y administrativa a partir de estudios de caso del sector eléctrico (Guerrero, 2004).

No obstante, se ha explorado muy poco el concepto de gobernanza transfronteriza desde el ámbito regional, es decir, más allá de las fronteras nacionales y de los mecanismos de integración subregional. En este sentido, este trabajo pretende contribuir a entender desde una perspectiva teórica las nuevas dinámicas regionales, las estrategias corporativas y el potencial de las redes de gobernanza desplegadas por una empresa colombiana que decidió rebasar las fronteras nacionales y penetrar mercados regionales mediante un sistema de infraestructura lineal.

En efecto, la concepción, implementación y gestión de una red de interconexión eléctrica de más de $35.000 \mathrm{~km}$ de línea que atraviesan cuatro países de la región, es un antecedente significativo de integración e interconexión en una región ligada a fórmulas institucionales de integración con limitado compromiso político.

\section{IMPLICACIONES TEÓRICAS Y MIRADAS A LA GOBERNANZA TRANSFRONTERIZA A NIVEL INTERNACIONAL}

La comprensión teórica y contextualización práctica del debate sobre la gobernanza transfronteriza exigen abordar el debate sobre la gobernanza planteado principalmente en la Unión Europea UE como resultado de su proceso de integración bajo una concepción espacial del Estado, coordinaciones público-privadas, alianzas intersectoriales y emergencias de nuevos tipos de asociaciones económicas entre fronteras.

La literatura sobre el elusivo concepto de gobernanza distingue dos marcos del concepto, diferenciándolo con claridad del de gobierno, caracterizado por estructuras jerárquicas de decisión del ámbito público. En primer lugar, se define como el proceso por el cual los actores públicos y privados se empeñan en una regulación de las relaciones y los conflictos sociales. En segundo lugar, como la representación teórica o conceptual de la coordinación de sistemas sociales. Finalmente, se refiere a la coordinación formal o informal de varios tipos de interacción público-privada bajo diferentes redes de asociación (Pierre, 2000).

Frente a estos dos marcos conceptuales se añade una tercera referencia normativa, relativa al buen gobierno ${ }^{1}$. Esta noción ha llegado a cumplir un papel protagónico en los discursos sobre

1. Término con el que los autores se refieren al concepto de good governance. 
desarrollo económico desde la década de 1980. El Banco Mundial y la Organización para la Cooperación Económica y el Desarrollo -Oecde- han defendido una administración fiscal prudente y eficiencia administrativa como precondición del desarrollo y el crecimiento sostenible, bajo un esquema político democrático, el estado de derecho, la libre competencia y el papel de las organizaciones no gubernamentales (Banco Mundial, 1997).

En un plano internacional, el fin de la guerra fría y la tensión ideológica internacional elevaron las nociones de efectividad y eficiencia en la solución de problemas como los principios guía que direccionan un sistema internacional de gobernanza (Kohler-Koch, 1993). Esta nueva visión fue complementada por una emergente discusión sobre el papel de los actores privados, tales como las corporaciones transnacionales y las organizaciones no gubernamentales ONG, con una variedad de funciones de gobernanza en el sistema de regulación económica internacional (Büthe, 2004; Hall \& Biersteker, 2002).

En Europa la discusión se dio en el campo de la teoría del Estado, ampliando los modelos de decisión jerárquica por formas de cooperación horizontal, decisiones conjuntas en sistemas verticales de autoridades públicas y redes políticas de actores estatales y no estatales del Estado Cooperativo $^{2}$ (Manytz \& Scharp, 2005). Por el contrario, el debate en la academia tanto americana como británica estuvo fuertemente influido por cambios en la coordinación de políticas y giró en torno de la crisis financiera del Estado, las reformas administrativas del sector público y el desarrollo de nuevos sistemas de gobernanza bajo una visión ideológica favorable al libre mercado (Pierre \& Peters, 2000).

\subsection{La construcción social de la escala}

La primera aproximación al problema de la gobernanaza a nivel global la encontramos a través de una visión crítica que vino a discutir la litetatura existente hasta entonces sobre el tema de la globalización (Castells, 1989; Ruggie, 1993). Esta literatura dio lugar a un duradero debate dentro de la ciencia política y la geografía política, como lo es el proceso de construcción social de la escala, el cual está en un principio directamente vinculado a una interpretación crítica de la gobernanza y el neo-liberalismo. Esta corriente de pensamiento, que se remonta a los trabajos de Brenner y Harvey, cuestiona la literatura sobre los espacios de flujo, la disolución de la geografía, el fin de las fronteras y la supraterritorialidad que traía consigo la globalización.

En esta corriente, el tema de la escala y la gobernanza es retomado en escenarios caracterizados por la globalización y la integración de los mercados. Por ejemplo, Brenner (1999) plantea, en primer lugar, la existencia de ejes territoriales fijos o aglomeraciones urbanas regionales

2. Término con el que los autores se refieren al concepto de Cooperative State. 
relativamente inmóviles y caracterizadas por la preponderancia de regulaciones nacionales; en segundo lugar, critica la corriente neoliberal de la globalización. De este modo, su argumentación se orienta hacia la discusión sobre las vías por las cuales las ciudades y los estados contemporáneos están siendo re-territorializados y sometidos a un procedimiento de construcción social escalar. En suma, para esta teoría, la escala emerge como un espacio histórico de regulación y una arena para la transformación social, donde se solidifican las relaciones de poder existentes, se regulan formas de cooperación y definen estrategias de poder (Swyngedouw's, 1997; Lipietz, 1994).

En esta línea de análisis, el trabajo de Perkman (2003; 2007) establece aportes sustanciales en la reflexión sobre las características de la escala y los marcos de gobernanza transfronterizos en Europa. De hecho, su trabajo toma distancia de la discusión teórica e ideológica del marxismo acerca de la escala y las zonas económicas naturales reivindicadas por los ideólogos del mercado, para investigar las formas empíricas bajo las cuales se afianzan efectivamente las escalas en los territorios. Su trabajo muestra un escenario regional europeo construido por una serie de relaciones e intervenciones entre las instituciones de la Unión Europea y gobiernos descentralizados, envueltos en: redes de gobernanza, cooperación transfronteriza, acuerdos organizacionales, diseño local y discursos territoriales influenciados por la cooperación y no por interdependencias funcionales, determinismo económico o marcos administrativos. En suma, la apuesta conceptual de Perkman está en el plano de la cooperación transfronteriza definida como una más o menos institucionalizada colaboración entre autoridades subnacionales contiguas, bajo el liderazgo de la agencia pública (Perkman, 2003; 2007).

Simultáneamente, otro tipo de análisis críticos sobre la globalización enfatiza que las regiones fronterizas son ejemplos de micro-regionalismo y construcción escalar a nivel económico, político y social, donde las escalas emergentes y existentes ganaban densidad institucional y sus fuerzas sociales intentan desarrollar nuevos mecanismos de articulación o coordinación. Una característica central de este tipo de enfoques está en que la región no es tratada como un territorio económico natural y sí como un fenómeno emergente, socialmente constituido, en el cual diversos actores y fuerzas sociales están en disputa, desde los migrantes económicos hasta empresas legales e ilegales, estados y movimientos no gubernamentales. (Jessop, 2004).

De este modo, la política económica de la construcción social escalar responde a la necesidad del Estado de redefinir su rol como un ámbito de regulación, adoptando estrategias de lucha contra la acumulación contingente del capital a través de estrategias de regulación y mecanismo de gobernanza. Bajo esta concepción, los procesos económicos se arraigan dentro de organizaciones socialmente construidas y procesos institucionales en los cuales los modos específicos de regulación son siempre emergentes, evolutivos y sometidos a una multiplicidad de acciones en contextos específicos y selectivamente estratégicos (Jessop, 1995).

En efecto, este enfoque permite comprender la pluralidad y la fluidez de los espacios estatales a través de procesos de coordinación regional entre el sector público y el privado. Bajo esta pers- 
pectiva, el Estado es un sistema relativamente abierto, contradictorio, híbrido y emergente que está activamente en desarrollo y en un constante proceso de transformación (Jessop, 1990).

\subsection{La gobernanza multinivel e interactiva}

Al interior del escenario europeo, el gran aporte académico a los aspectos transfronterizos de la gobernanza provino de la discusión alrededor de la formulación de políticas y coordinación en la UE. Una característica central de la gobernanza en la UE es su naturaleza multinivel (Marks, 1993), en otras palabras, un sistema de continua negociación a través de varios niveles -local, nacional y regional- interconectados y no anidados de gobierno territorial.

La gobernanza multinivel postula que la autoridad para la toma de decisiones no está monopolizada por los gobiernos de los estados y es difundida de manera interdependiente a los diferentes niveles de toma de decisiones, bajo formas no jerárquicas a actores públicos como privados (Hooge \& Marks, 2001).

Por su parte, los sistemas de gobernanza interactiva se caracterizan por la interdependencia de actores públicos y privados actuando en diferentes niveles, donde la toma de decisiones tiende a no ser jerárquica. En un sistema de esta naturaleza, el rol del Estado cambia en relación a la autoridad tradicionalmente entendida como vertical, para reemplazarse por la figura de un activador o un socio estratégico mediante el uso de acuerdos que operan a través de reglamentaciones blandas -soft law- (Kholer-Koch \& Rittberger, 2006). Por consiguiente, la gobernanza interactiva en las sociedades contemporáneas se aborda en términos de la obtención de consensos mediante la negociación con diferentes actores sociales, enfatizando la solución de problemas y logrando acuerdos mediante el uso de normas e incentivos financieros emanados de fuentes gubernamentales legítimas (Kholer - Koch, 2004).

\subsection{El "ajuste"}

A partir de la noción sobre el "ajuste" (Gualini, 2003) se explora la posibilidad de constituir un programa de investigación empírica que tome distancia del "nacionalismo metodológico" y pueda contribuir al avance del conocimiento y a la refinación teórica de los procesos relacionados con la política europea multinivel. El trabajo de Gualini, a través del caso italiano, reivindica la fuerza de la gobernanza multinivel en contra de una noción estado-céntrica y como una plataforma intermedia de innovaciones institucionales eficientes para toda Europa (Gualini, 2003; 2007).

Este tipo de análisis, a diferencia de visiones más cooperativas, como las de Perkman, o de resistencias institucionales y organizacionales bajo esquemas de regulación, como las de 
Jessop, descritas antes, centra su estudio en las diversas y contradictorias trayectorias de procesos de cambio institucional, relacionados con la construcción de varios niveles de gobierno. En esta dirección, la gobernanza multinivel se presenta como un proceso de replanteamiento de relaciones intergubernamentales, atravesadas por una estructura que enfatiza en la emergencia de nuevas oportunidades y resistencias a compromisos institucionales que permitan superar los factores contradictorios y conflictivos del marco institucional y normativo (Gualini, 2007).

Otros trabajos han tomado mayor distancia del nacionalismo metodológico y del sesgo político implícito en la metodología escalar, desafiando las nociones jerárquicas de escala y toda noción territorial mediante una serie de estudios sobre el tema fronterizo y regional en Europa (Deas \& Lord, 2006), que ilustran el vasto potencial de cambio de la geografía política y económica europea sobre las fronteras y el nacionalismo como el más formidable obstáculo para la integración. Bajo esta dimensión geoeconómica, las regiones transfronterizas son asumidas como unidades imaginadas de competencia global y optimización de la aglomeración en b usca del desarrollo económico (Johnson, 2009). Finalmente, el trabajo afirma que la regionalización en esta parte de la UE -la región de Sajonia- es mediada y activamente cooptada por actores locales y regionales para sus propios propósitos, en medio de un conjunto de instituciones y redes de gobernanza implementadas en las regiones por los gobiernos locales y estatales, las agencias de desarrollo económico y las ONG (Johnson, 2009).

\subsection{El nuevo regionalismo}

La reflexión y reivindicación analítica de lo regional al tomar abierta distancia del enfoque de construcción social de la escala, plantea el estudio de lo regional a través de una concepción de lugares abiertos, discontinuos, relacionales e internamente diversos (Allen, 1996). Esta alternativa espacial piensa la región como una red ensanchada de relaciones sociales que "revela no un área" (Amin, 2003) propiamente, pero sí un complejo entramado de relaciones desarticuladas. Las ciudades y regiones son, por consiguiente, interpretadas como sitios dentro de redes de variada composición geográfica y espacios de movimiento y circulación de bienes, tecnologías, conocimientos, personas, finanzas e información (Amin, 2003).

Desde la perspectiva del "nuevo regionalismo", inspirado en los trabajos de los geógrafos californianos Allen Scott (1998) y Michael Storper (1995; 1997), se plantea un desafío a la perspectiva territorial. Los desarrollos de este enfoque plantean una configuración topológica del espacio, constituido como un entramado de redes de trabajo y conexiones relacionales, que no son fijadas o localizadas en un lugar, pero sí son constituidas a través de varias "entidades circulantes" coreografiadas a través de conexiones topológicas transterritoriales, esferas públicas virtuales y trazos de asociación rizomáticos (Amin, 2004; Barry, 2001; Latour, 1999; 2005; Urry, 
2003). Bajo este enfoque se aborda el consenso institucional a partir de una variedad de organizaciones público-privadas y organizaciones intermediarias en una región constituida por relaciones heterárquicas (Amin, 2003).

En conclusión, el "nuevo regionalismo" enfatiza en los procesos de convergencia a través de la constitución de firmas, clusters y redes de proveedores en nuevos espacios industriales o complejos de producción territorial. Bajo este enfoque, las investigaciones señalan el papel crucial desempeñado por nuevas asociaciones económicas en regiones como Emilia Romagna y BadenWurtemberg a través de intervenciones público-privadas en infraestructuras de información, formación de capital humano y transferencia tecnológica; junto a la consolidación del espesor institucional a través de valores codificados, conocimiento tácito y agendas regionales comunes (Cooke \& Morgan, 1998; Amin \& Thrift, 1994).

Finalmente, dentro del análisis espacial en geografía las redes constituyen sistemas arteriales de organización regional que posibilitan la circulación de flujos. La noción de "red" descansa en conceptos de diversidad y heterogeneidad territoriales en la distribución de puntos de producción y consumo de bienes y servicios y, en discontinuidades temporales y espaciales, que conllevan su necesaria eliminación a través del intercambio. En este sentido, es en la interrelación establecida entre focos de generación y atracción entre canales de circulación y flujos (líneas) donde encontramos la noción de red o circuito (Seguí, 1994).

En conclusión, las diferentes aproximaciones de la literatura varían dependiendo de sus marcos teóricos, casos empíricos y diferencias de enfoque. No obstante, hay un amplio consenso sobre la pertinencia analítica y metodológica del concepto de gobernanza, con algunas excepciones que lo descartan de tajo reduciéndolo en el mejor de los casos a una metáfora descriptiva del mundo contemporáneo (Keating, 2004). Ahora bien, más allá de los debates teóricos, la aplicación práctica del concepto de gobernanza multinivel en la Unión Europea ofrece interesantes resultados que permiten comprobar las dinámicas subyacentes y los horizontes del proceso a nivel social e institucional.

En este sentido, las investigaciones que abordan el problema de Europa y sus regiones muestran que la Comisión Europea ha diseñado marcos y métodos que pretenden estimular el potencial endógeno de las regiones y sus empresas, la innovación tecnológica, la provisión de servicios avanzados y la construcción de infraestructuras modernas (Tömmel, 2004).

Las regiones se han transformado en el sentido de perseguir sus intereses de modo independiente y activo. Iniciativas comunitarias y planes piloto gestionados por la Comisión, junto a la implementación de novedosas propuestas, como la iniciática comunitaria del Fondo Europeo de Desarrollo Regional Interreg, han servido para fomentar la cooperación transfronteriza, la transferencia de conceptos y el intercambio de experiencias entre regiones. En este sentido, actores 
no gubernamentales, como asociaciones y consorcios empresariales, centros de negocios y agencias de desarrollo regional, han subsidiado a través de mecanismos, como la subvención global a pequeñas y medianas empresas y a otros actores descentralizados, la creación y fortalecimiento de redes europeas. A largo plazo, estas estrategias de la UE sugieren la transferencia de poderes, tareas y funciones genuinamente públicas a agentes privados, así como la transformación de relaciones entre instituciones públicas y actores privados (Tömmel, 2004).

En conclusión, podemos afirmar que el caso europeo, a la luz de la discusión interdisciplinar sobre la gobernanza multinivel, la construcción social de la escala, las redes de gobernanza y el nuevo regionalismo, sugiere la aproximación a una nueva compresión y representación del espacio a través de redes de interconexión gestionadas por medio de un modelo coordinado entre el sector público y el privado. Por consiguiente, es importante explorar el tema y aportar al debate a través de un caso de gobernanza transfronteriza y gestión de integración energética como el de ISA.

\section{ISA: UN CASO DE REDES DE GOBERNANZA TRANSFRONTERIZA EN UN ESPACIO REGIONAL}

A continuación estudiamos el caso de la empresa colombiana ISA Interconexión Eléctrica S.A., la cual nace en Medellín, Antioquia, en 1968, y es hoy una de las empresas más reconocidas en el sector eléctrico que busca en los próximos 5 años estar entre las tres empresas más grandes de América y la más grande de Latinoamérica. El modelo ISA, que a través de sus filiales y subsidiarias adelanta importantes proyectos de infraestructura lineal que impulsan el desarrollo en el continente, puede ser estudiado como un modelo de gobernanza que logró rebasar las fronteras nacionales.

\subsection{Antecedentes y modelo de análisis}

El proyecto de ampliación y consolidación de una infraestructura lineal de punto a punto por parte de la empresa colombiana ISA Interconexión Eléctrica S.A., es hoy una experiencia y un modelo de gestión y gobernanza de alcance internacional que, gracias a la consolidación de su proyecto de infraestructura eléctrica superior a los 35 mil kilómetros en más de cuatro países del subcontinente, se ha convertido en un antecedente y un modelo de interconexión regional. En este sentido ISA ha gestionado su modelo por medio del cambio y la transformación del escenario regional, prescindiendo de los medios tradicionales de autoridad a través de una serie de implicaciones y oferta de canales de acceso técnico, participación accionaria, incorporación de principios de gobernanza y gobierno corporativo, propagación de conceptos de gestión y una visión regional que ha generado cambios en los patrones de interacción y en la composición de las redes en escala transnacional. 
Si bien los factores más determinantes en el éxito empresarial de ISA pueden atribuirse a características propias de su gestión, estrategia y visión, sus experiencias y logros pueden ser interpretados bajo una mirada típicamente política y como un claro antecedente de gobernanza y proyección exitosa hacia la integración en la región. Es así como, siguiendo a Susan Strange (2001), se puede ampliar la definición de la política más allá de los estados, para abarcar a todas las fuentes de autoridad con poder para asignar valores -seguridad, riqueza, justicia y libertad-. En cierto sentido, este desplazamiento de autoridad de los estados a los mercados ha transformado a las empresas transnacionales en actores políticos.

La experiencia de ISA debe pensarse al interior del proceso de privatización del sector eléctrico regional entrados los años 1990, cuyo objetivo central fue el de una mayor competitividad en el proceso de generación, transmisión y distribución eléctrica, siguiendo el modelo adoptado en el Reino Unido, buscando desestimular la monopolización del sector eléctrico que para entonces se encontraba en manos del Estado. En esos años el sector eléctrico colombiano se caracterizaba por el subsidio de las tarifas, la politización de las empresas estatales y los altos sobrecostos y atrasos generados por las altas inversiones en infraestructura que eran una costosa carga para el Estado (Guerrero, 2004).

Colombia privatizó entre 1992 y 1998 un total de US \$ 5.196 millones en un sistema de 12 GW, frente a otros países de la región que, como Brasil, en el mismo periodo privatizaron un valor de US \$21.023 millones en un sistema de 62 GW (Guerrero, 2004).

En el proceso de apertura y atracción de capitales privados en la región se intentó fortalecer a los mecanismos legales para proteger los intereses de los consumidores, el Estado y los agentes privados que asumieron el riesgo de invertir y administrar la operación energética. Para ello se utilizaron estrategias de gobernanza que promovieron la creación de un ambiente de colaboración y cooperación entre el sector público y el sector privado, fundamentales en la obtención de un escenario de mercado que permitiera fijar precios, costos y mecanismos de financiamiento.

Se puede afirmar que la reforma del sector eléctrico colombiano se fundamentó en la reorientación del Estado hacia actividades que le fueran propias, así como en la redefinición de actividades productivas que pasaron de manos del Estado a ser administradas con criterios de administración privada de eficiencia y expansión de mercados.

Ahora bien, la estrategia de análisis metodológico del caso se construye a partir de la selección de algunos de los aportes de la literatura sobre gobernanza en Europa, para establecer un marco que permita identificar y organizar las características centrales del modelo de gobernanza interactiva de la empresa a partir de las estrategias centrales de la concepción del modelo, las interconexiones establecidas por la red de infraestructura lineal y las innovaciones a que dio lugar la concepción y consolidación del proyecto ISA. Sobre estas tres variables se identifican tres momentos correspondientes que buscan facilitar la visualización temporal del proceso: 
apertura, diseño y consolidación del modelo. El análisis del caso se centra en las estrategias gestionadas y coordinadas por la empresa desde su centro de operaciones en Medellín, ciudad donde se realizaron las encuestas con altos directivos y se recabó la información referente al proyecto de expansión nacional y regional, sin entrar a detallar los desarrollos propiamente técnicos de la red, las alianzas y apropiaciones del modelo por parte de otros estados en la región y filiales de la empresa en los diferentes países donde ISA ha consolidado su red de infraestructura.

\subsection{El Modelo ISA}

Por su parte, la empresa ISA ha sido una abanderada en diferentes foros y escenarios internacionales del tema de la integración física de los mercados eléctricos en la región. En este sentido, la empresa emprendió un proceso de mejoramiento y ampliación de su sistema eléctrico de intercambio buscando el máximo aprovechamiento de la capacidad energética de todo el sistema.

Durante los años 1990 quedó en evidencia el desequilibrio entre el nivel de potencia eléctrica alcanzado al interior de cada país frente al sistema eléctrico interestatal y de los países fronterizos, donde los sistemas nacionales resultaban adecuados únicamente a las necesidades circunscritas a la demanda de sus espacios territoriales. De esta manera, quedaba claro que los notables beneficios de la asociación y las alianzas eléctricas estaban siendo desaprovechados en la región. De hecho, algunos estudios han señalado los problemas internos de gobernanza y falta de perspectiva regional que han padecido los sistemas de transmisión eléctrica regionales, desencadenando crisis consecutivas de abastecimiento energético en toda la región como lo evidenciaron los casos de desabastecimiento energético en Brasil y Argentina en el año 2001, y en Bolivia en el año 2003 y 2004 (Botto, 2007).

Fue así como, tras el proceso de separación entre ISA e ISAGEN, la empresa se enfocó y especializó en el transporte de energía en un sector con ingresos regulados y crecimiento vegetativo atado al crecimiento nacional. Así, en la década de los 90, de cara a un Sistema de Transmisión Nacional STN con pocas oportunidades de conexiones y ampliación de sus mercados, la empresa decidió mirar hacia América del Sur, pues hasta entonces el esquema de regulación colombiana limitaba a la empresa a las licitaciones de la Unidad de Planeación Minero Energética UPME, adscrita al Ministerio de Minas y Energía. En este sentido, el proceso de expansión e integración regional fue producto de la estrategia de crecimiento dentro de la visión empresarial del grupo, siempre respondiendo a la confianza que los accionistas habían puesto en el futuro financiero de la empresa. En esa dirección surgieron los temas de interconexiones, compra de redes en otros países de la región, participación en convocatorias y licitaciones internacionales, gerenciamiento de proyectos y operación de mercados dentro de una visión de negocios de infraestructura lineal punto a punto. 
En este punto, la convergencia en los cambios regulatorios a nivel nacional y regional, en un escenario nacional donde la empresa afrontaba restricciones a su crecimiento y diversificación empresarial, llevaron a ISA a ingresar en los mercados regionales buscando oportunidades de expansión en la integración física de los mercados eléctricos. Para sus directivos, si bien la integración física es aún limitada, donde esta se ha presentado, ISA ha sido pionera en su consolidación.

En consecuencia, el objetivo de la interconexión eléctrica y la integración regional a través de sistemas eléctricos ha estado en que el benéfico neto de las redes eléctricas nacionales sea superado por los benéficos obtenidos a nivel regional. En este punto se encuentra hoy en día la empresa ISA, la cual controla el 75\% del mercado de transmisión eléctrica nacional y obtiene el 55 \% de sus ingresos del Brasil y el 37 \% del mercado colombiano (Valores Bancolombia). El escenario alcanzado es más que revelador y en parte responde a los interrogantes que hace una década se planteaban sobre los limitados avances en la interconexión de la región. El avance de ISA demuestra las posibilidades y potencialidades del sector eléctrico en la integración regional. Este potencial del sector eléctrico ya ha sido identificado a nivel regional por su importante papel en el intercambio de experiencias y aprovechamiento de externalidades entre países a través de sistemas regionales de innovación y en la construcción de infraestructuras y redes de comercialización de servicios energéticos por fuera de estructuras organizacionales adicionales (Rosenwurcel, G; Bezchinsky, G; Gianello, C \& Thomas, H, 2008). Este estudio viene a reafirmarse por los directivos de la empresa, quienes confirman cómo a través de "la importación de buenas prácticas" con diferentes empresas del sector se consiguió una "verdadera integración regional".

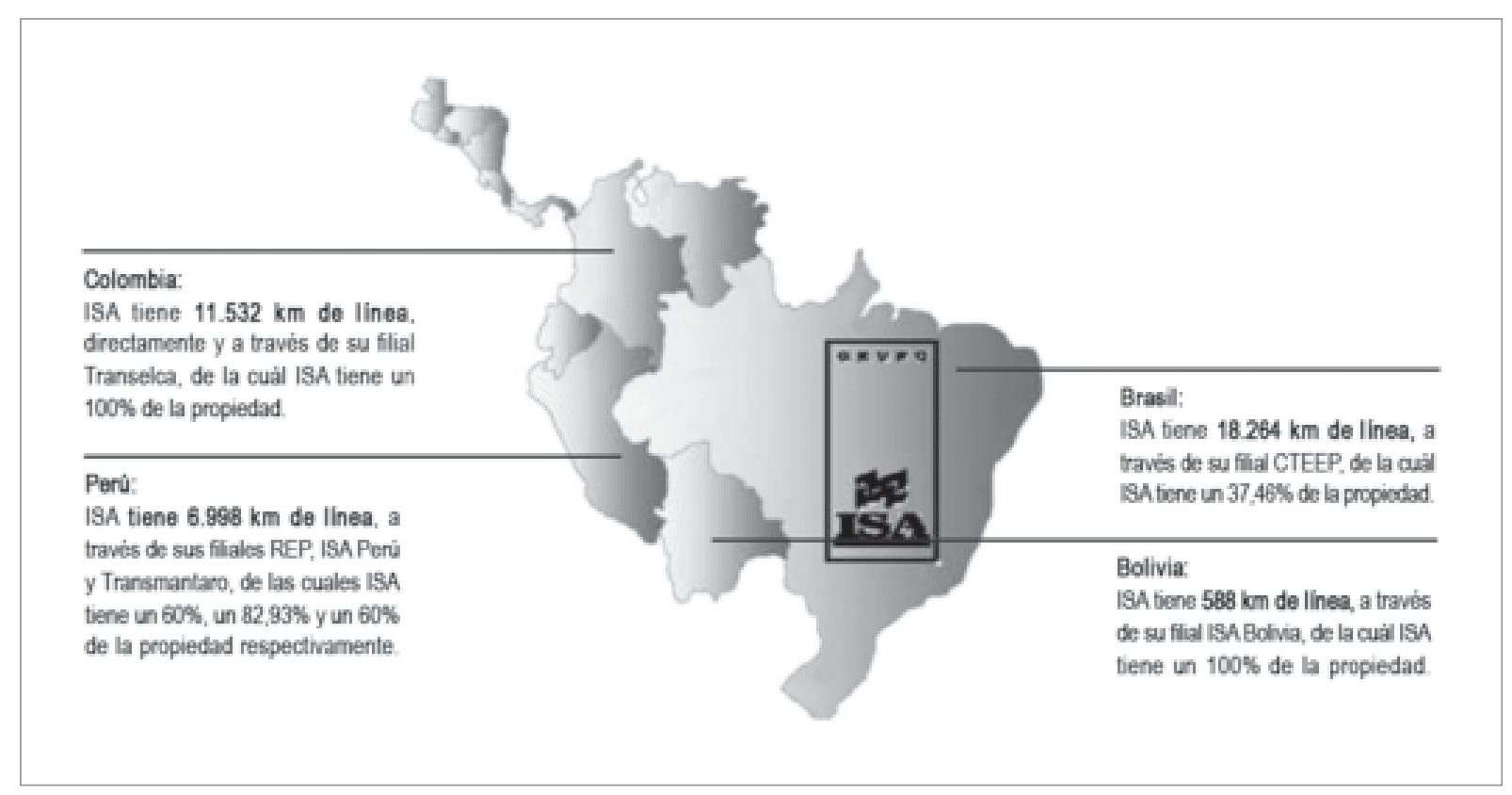

Fuente: ISA. 
En el caso de ISA, la empresa administra junto a sus filiales en la región 37.382 km de línea; su sistema de infraestructura lineal de punto a punto es asimilado por la empresa como un sistema de interconexión fluvial que tiene como objetivo transportar energía, personas, datos y telecomunicaciones con el ánimo de promover el desarrollo y el movimiento social de las personas y las poblaciones por las cuales avanza su sistema de interconexión ISA. La empresa, a través de tres grandes emisiones, fue una de las primeras empresas colombianas en incursionar en la bolsa de valores y tiene uno de los mayores números de accionistas, con lo cual consiguió su objetivo de democratizar la propiedad accionaria, vincular capital privado, consolidar su bursatilidad y contribuir a su fortalecimiento, expansión y crecimiento. La acción de ISA se ha consolidado como una de las de mayor liquidez y negociabilidad en la bolsa, el precio reportado por Bancolombia para el cierre del año 2010 era de \$ 13.800 pesos frente a \$ 7.000 pesos que reportaba en enero de 2009 .

\section{El Modelo ISA:}

\begin{tabular}{|c|c|c|}
\hline Apertura & Diseño & Consolidación \\
\hline Estrategias & Conexiones & Innovación \\
\hline $\begin{array}{l}\text { - Visión regional. } \\
\text { - Democratización } \\
\text { accionaria. } \\
\text { - Gobierno } \\
\text { corporativo. }\end{array}$ & $\begin{array}{l}\text { Interconexión regional: } \\
\text { - Perú } \\
\text { - Bolivia } \\
\text { - Ecuador } \\
\text { - Brasil }\end{array}$ & $\begin{array}{l}\text { - Infraestructra lineal } \\
\text { - } \text { Impunto a punto. } \\
\text { nas prácticas. } \\
\text { - Sinergias estratégicas } \\
\text { con proveedores y } \\
\text { firmas contratistas. }\end{array}$ \\
\hline
\end{tabular}

La democratización accionaria de ISA fue una pieza estratégica dentro de su proceso de expansión y en el afianazamiento de su marco de gobernanza corporativa con el Gobierno colombiano. En primer lugar, en su proceso de proyección definió su visión, estrategias y tareas para penetrar en un competitivo mercado regional, asumiendo que debía establecer una relación mucho más eficiente con los mercados financieros. Por este motivo decidió tomar distancia del modelo con el cual históricamente se había financiado a través de recursos de organismos multilaterales en el viejo esquema del sector eléctrico y buscar la democratización accionaria de la empresa. En segundo lugar, en la relación con el Gobierno colombiano como socio mayoritario de la empresa se ha respetado la autonomía del gobierno corporativo y mantenido las prácticas corporativas de la empresa al interior de la Junta Directiva.

El proceso de consolidación de la empresa y sus filiales en un plano internacional obedece a un exitoso proceso de gestión privada y gobernanza transfronteriza en un escenario regional. 
La empresa ganó la Licitación Pública Internacional del Gobierno Peruano sobre los sistemas de transmisión eléctrica del Restado (Etecen-Etesur). Con esta adquisición la empresa se convirtió en el mayor transportador de energía eléctrica del Perú. A finales de 2002, las compañías Internexa de Colombia y Transelectric de Ecuador dieron origen a la empresa Transnexa, encargada de administrar la red de fibra óptica que une a Colombia con Ecuador, e interconecta a nuestro vecino con el mundo. En el año 2003, la empresa ganó en La Paz la licitación para construir y operar durante 30 años cinco subestaciones y tres líneas de transmisión de energía en Bolivia, consolidando así su objetivo de expansión en la Comunidad Andina (Guerrero 2004).

Los logros alcanzados en la interconexión eléctrica entre Ecuador, Perú y Colombia dieron impulso y sentaron las bases del proceso de interconexión eléctrica en la región, el cual fue ratificado con las firma de la Declaración de México, en marzo de 2001, y con la suscripción en Cartagena del "Acuerdo para la Interconexión Regional de los Sistemas Eléctricos y el Intercambio Internacional de Energía Eléctrica. Fue así como, gracias a la coordinación y exitosos procesos de gobernanza regional, se obtuvo en diciembre de 2002 la Decisión 536 por parte de la Comunidad Andina CAN sobre la Integración Regional de los Sistemas Eléctricos de la Comunidad Andina. De este modo, para la CAN "la interconexión de los sistemas eléctricos de los países miembros y los intercambios comerciales intracomunitarios de electricidad pueden brindar importantes beneficios a los países miembros en términos económicos, sociales y ambientales y pueden conducir a la utilización óptima de sus recursos energéticos y a la seguridad y confiabilidad en el suministro eléctrico" CAN.

Más recientemente, la entrada de ISA al Mercosur tuvo en principio como soporte el Acuerdo Marco para la Creación de la Zona de Libre Comercio entre el Mercosur y la Comunidad Andina el 16 de abril de 1998, donde se propuso promover el desarrollo y la utilización de infraestructura física y energética para reducir costos y promover la competitividad regional.

En el proceso de entrada al Brasil durante el año 2006 ISA adquirió en principio el 50\% de las acciones con derecho a voto de la Cteep (Companhia de Transmissao de Energía Eléctrica Paulista) y posteriormente su participación accionaria aumentó a un 89\% a través de una licitación regulada por la Agencia Nacional de Energía Eléctrica Aneel del Brasil. De este modo, a través de la Cteep la empresa ISA opera el $10 \%$ de las redes de electricidad que comprenden el BSIN (principal sistema interconectado del Brasil). La Cteep es actualmente considerada como una de las mejores compañías del sector eléctrico en Brasil, la cual transporta casi la totalidad de la energía que se consume en el estado de Sao Paulo, el 30\% de toda la energía eléctrica que se produce en el país y el $60 \%$ de la energía que se consume en el sudeste del país. Esta adjudicación, sumada a una serie de licitaciones exitosas por parte de la empresa, demuestra el potencial de negociación y gobernanza que ISA ha sabido desplegar a nivel regional. Dentro de este exitoso proceso de expansión, una dimensión fundamental han sido sus fortalezas en los previos estudios financieros, técnicos y legales, valoradas por sus altos ejecutivos como piezas fundamentales dentro de los positivos resultados licitatorios que la empresa ha obtenido. 
En particular, este exitoso proceso de licitación se realizó a través de la constitución de un vehículo de inversión: ISA Capital do Brasil, Ltda., con sede en la ciudad de Sao Paulo, que fue transformado en sociedad anónima de capital abierto. La licitación pública, realizada en la Bolsa de Valores de Sao Paulo, adjudicó a ISA a través de esta sociedad inversionista el control accionario de una de las compañías públicas más emblemáticas del sector eléctrico brasilero, como la Cteep, a través de una operación cercana a los US \$ 887 millones, lo cual le permitió el control del $89 \%$ de las acciones ordinarias ISA.

La empresa ha establecido una sinergia estratégica con sus empresas proveedoras y firmas contratistas en el sector de la construcción e ingeniería para penetrar en los mercados regionales. En tal sentido, la penetración en otros países se ha conseguido de la mano de las alianzas establecidas con estas empresas que habían obtenido buenos resultados y ganado posiciones y conocimientos estratégicos en los mercados regionales, para fortalecerse y acompañarse en el posicionamiento regional por medio de sinergias de información, buenas prácticas y redes de posicionamiento estratégicas.

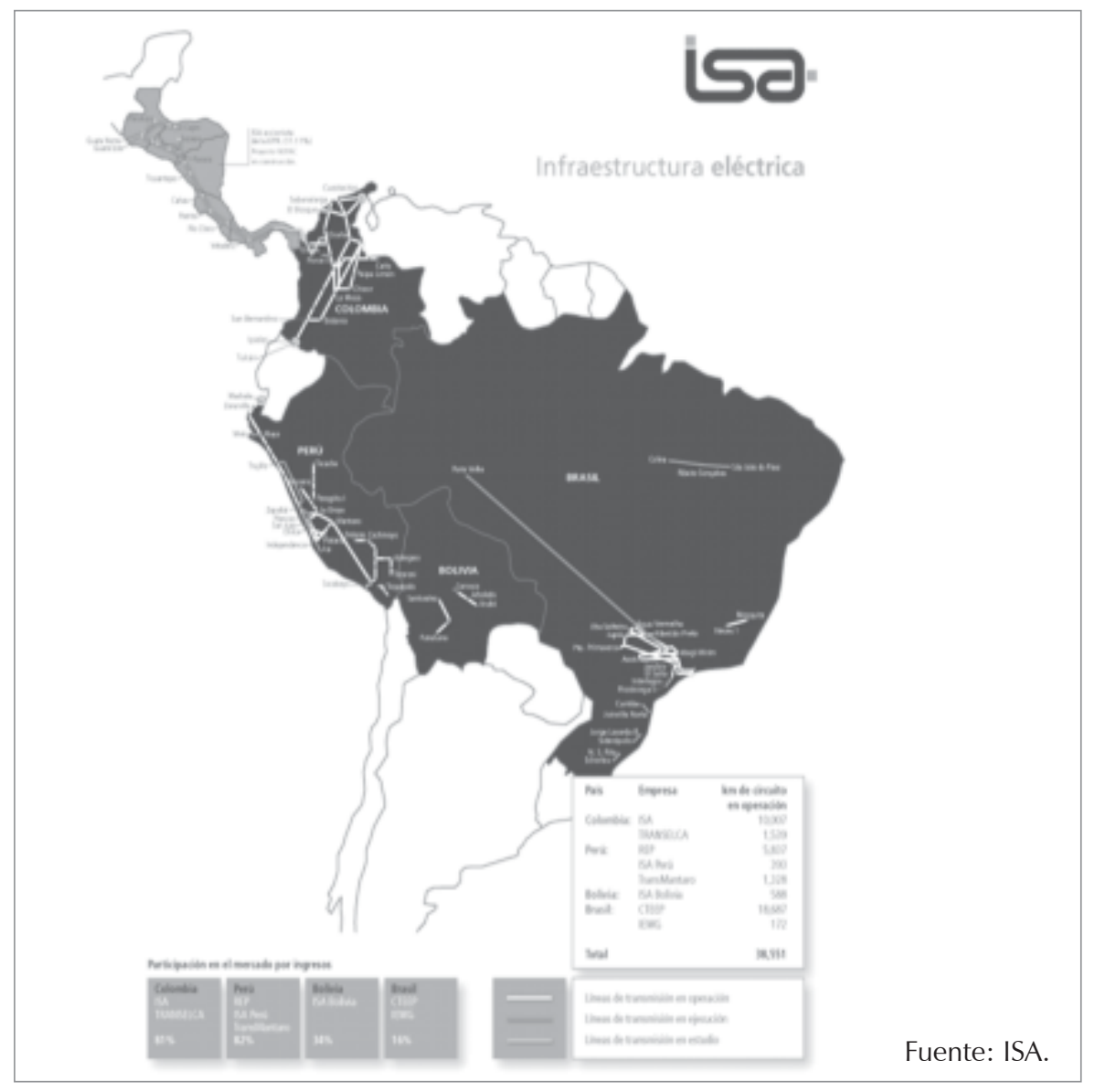


Es así como la estrategia de ISA ha alcanzado exitosos estándares a nivel regional, gracias a los acuerdos y asociaciones promovidas entre gobiernos, instituciones y empresas, lo que le ha permitido construir y gestionar un sistema de interconexión eficiente a escala regional. Su estrategia ha estado en la consecución de adquisiciones, concesiones e interconexiones internacionales y en la prestación de servicios e integración de mercados a través de infraestructuras energéticas punto a punto. Por consiguiente, su modelo puede analizarse como una exitosa experiencia y poderosa transformación en la representación espacial de la región, convirtiéndose así en un notable antecedente y modelo de negociación y gestión dentro del proceso de integración regional del subcontinente.

\section{CONCLUSIONES}

La exploración y discusión teórica sobre la gobernanza transfronteriza cobra cada día mayor pertinencia en la región de cara a las transformaciones económicas y proyecciones que líderes políticos y actores privados llevan a cabo a través de estrategias coordinadas y visiones empresariales de alcance global.

Este estudio aporta mayor claridad al universo teórico de la gobernanza, mediante el análisis del modelo ISA y su relación con el entorno regional y con las dinámicas público-privadas de actores locales. De hecho, el énfasis de la investigación está en estas nuevas constelaciones regionales, producto de diferentes modalidades de gobernanza, prácticas administrativas y visiones transfronterizas que producen cambios concretos y alteran la forma como entendemos la integración y el desarrollo regional, más allá de la discusión sobre las políticas de integración bajo la dirección exclusiva de los estados y cancillerías en la región.

El modelo de gobernanza transfronteriza desplegado por la empresa ISA, caracterizado por una visión espacial regional, gobierno corporativo, democratizacion accionaria, transferencias de tecnología, buenas prácticas, fortalezas financieras, técnicas y legales y su gestión de un sistema de alcance regional, ha logrado rebasar de manera perdurable las fronteras territoriales, los marcos institucionales y las experiencias previas de que disponíamos para analizar y proyectar el problema de la integración regional. Por ello, el caso de ISA contribuye al análisis de las redes de gobernanza transfronteriza y a replantear los retos que la región tiene a futuro en la consolidación de su integración e interconexión.

Finalmente, la investigación sobre gobernanza puede contribuir a una mayor comprensión, aplicación y transformación de las políticas públicas a nivel nacional y regional. Además, esta contribuye a explorar las condiciones tanto complejas como cambiantes de la relación entre el sector público y el privado, y a evaluar experiencias de transformación regional que permitan identificar nuevas posibilidades y retos que la región tiene con el futuro. 


\section{BIBLIOGRAFÍA}

- Agnew, J. (1994). The territorial trap: The geographical assumption of international relations theory, Review of International Political Economy.

- $\quad$ Allen, D. (1996). Cohesion and Srtuctural Adjustmente. En Wallace, H. \& Wallace, W. (Eds.) Policy Making in the European Union. (p. 209-233). Oxford: Oxford University Press.

- Amin, A. (1999). An institutional perspective on regional economic development. International Journal of Urban and Regional Research, 23, 365-378.

- Amin, A. (2003). Lost Geographies of Power. Oxford: Blackwell.

- Amin, A. (2004). Regions unbound: towards a new politics of place. Geografiska Annaler, 86, 33-44.

- Barry, A. (2001). Political Machines: Governing a Technological Society. London: Athlone.

- Beck, U. (2004). Poder y contrapoder en la era global. La nueva economía política mundial. Barcelona: Paidos.

- Beltrán, L. (2006). Dinamismo comercial pese a las diferencias políticas. En Socorro, R. \& Cárdenas, J. (Cords.) Colombia-Venezuela Retos de la convivencia. (p.75-92). Bogotá: Unibiblos.

- Botto, M. (2007). Saber y Política en América Latina. El uso del conocimiento en las negociaciones comerciales internacionales. Buenos Aires: Prometeo.

- Brenner, N. (1999). Globalization as Reterritorializacion: The Re-scaling of Urban Governance in the European Union. Urban Studies, 36, 431-455.

- Büthe, T. (2004). Governance through Private authority: Non-State Actors in World Politics, Journal of International Affairs 58: 281-290.

- Castells, M. (1989). La era de la información: economía, sociedad y cultura. Madrid: Alianza Editorial.

- Cooke, P. \& Morgan, K. (1998). The associational Economy: Firms, Regions, and innovation. Oxford: Oxford University Press.

- Deas, I \& Lord, A. (2006). From a new regional to an unusual regionalism? The emergence of nonstandard regional spaces and lessons for the territorial reorganization of the state. Urban Studies, 43, 1847-1877.

- García, A. \& Urdaneta, A. (1999). Desarrollo Fronterizo: Imperativo binacional. Colombia y Venezuela: Proyección internacional y relación bilateral. En Ramírez, S. \& Cárdenas, J. (Cords). Colombia Venezuela. Agenda Común para el Siglo XXI. (p. 281-306). Santa Fe de Bogotá: Tercer Mundo. 
- Guerrero, B. (2004). La globalización y su efecto en el sector eléctrico latinoamericano. -Caso interconexión eléctrica S. A. - ISA-. Tesis de Maestría en Administración de la Universidad de los Andes. Bogotá.

- $\quad$ Ghemawat, P. (2008). Redefiniendo la Globalización. Barcelona: Deusto.

- Gilpin, R. (2003). El reto del Capitalismo Global: la Economía mundial en el siglo XXI. Madrid: OcéanoTurner.

- Goldin, I. \& REINHERT K. (2005). Globalización y Pobreza. Bogotá: Banco Mundial.

- Gualini, E. (2003). Challenges to multi-level Governance contradictions and conflicts in the Europeanization of Italian regional policy. Journal of European Public Policy, 10, 616-634.

- Gualini, E. (2004). Regionalization as "Experimental Regionalism": The Rescaling of Territorial Policymaking in Germany. International Journal of Urban and Regional Research 28, 329-353.

- Gualini, E. (2006). The Rescaling of Governance in Europe: New Spatial and Institutional Rationales. European Planning Studies, 14, 881-904.

- Hall, R. \& Biersteker, T. (2002). The emergence of Private Authority in Global Governance. New York: Cambridge University Press.

- Harvey, D. (1983). Teorías, leyes y modelo en geografía. Madrid: Alianza Editorial.

- Held, D. (1997). La democracia y el orden global. Del estado moderno al gobierno cosmopolita. Barcelona: Paidós.

- Hooghe, L. \& Marks, G. (2001). Multi-Level Governance and European Integration. Lanham: Rowman \& Littlefield.

- Jessop, B. (2004). La economía política de la escala y la construcción de las regiones transfronterizas. EURE, 89, 26-41.

- Jessop, B. (1995). The regulation approach, governance and post-fordism: alternative, perspectives on economic and political change? Economy and Society, 24, 307-333.

- Johnson, C. (2009). Cross-border regions and territorial restructuring in central Europe room for more transboundary SPACE. European Urban and Regional Studies, 16, 177-191.

- Keating, M. (2004). La política territorial y el nuevo regionalismo. En: Gobernanza multinivel en la Unión Europea, Ed. Francesc Morata, 189-217, Valencia: Tirant Lo Blanch.

- $\quad$ kholer-koch, B. \& Rittberger, B. (2006). The "Governance turn" EU Studies. Journal of Common Market Studies, 44, 27-49. 
- Kholer-koch, B. (2004). Gobernanza interactiva: las regiones en la red de la política europea. En Francesc Morata (Ed.) Gobernanza multinivel en la Unión Europea. (p. 121-158). Valencia: Tirant Lo Blanch.

- Latour, B. (1999). On rescaling ANT. En John, H. (Ed.) Actor Network Theory and After. (p.15-25). Oxford: Blackwell.

- Lipitez, A. (1994). The national and the regional: Their autonomy vis-a-vis the Capitalist World Crisis. En Ronen, P. \& Barry, G. (Eds.) Trascending the State-Global Divide. (p. 24-43). Boulder: Lyann Rienner.

- Londoño, J. (2008). Valor y precio de las exportaciones colombianas a Venezuela entre 1999-2007 frente a tres países de referencia. Borradores de Economía, Banco de la República, 516.

- Macleod, G. \& Jones, M. (2007). Territorial, Scalar, Networked, Connected: In What Sense a "Regional World"? Regional Studies, 419, 1177-1191.

- Mayntz, R. \& Scharp, F. (1995). Policy-Making in the German Federal bureaucracy. Amsterdam: Elsevier.

- Marks, G. (1993). Structural Policy and Multilevel Governance in the EC. En Cafruny, A. \& Rosenthal, G. (Eds.) The State of the European Community. Boulder: Lynne Rienner.

- Mojica, A. \& Paredes, J. (2004). La economía colombo- venezolana y su impacto en la región de frontera. 1999 - 2003. Bucaramanga: Centro Regional de Estudios Económicos.

- Montes, E., Garavito, A. \& Pulido C. (2008). El mercado venezolano en las exportaciones colombianas: dependencia de los exportadores en 2006. Borradores de Economía, Banco de la República, 492.

- Perkmann, M. (2003). Cross-border regions in Europe - Significance and drivers of regional cross-border co-operation. European Urban and Regional Studies, 10, 153-171.

- Perkmann, M. (2007). Construction of New Territorial Scales: A Framework and Case Study of the EUREGIO Cross-border Region. Regional Studies, 41, 253-266.

- Pierre, J. (2000). Introduction: Understanding Governance. En Pierre, J. (Ed.) Debating Governance, Authority, Steering and Democracy. Oxford: Oxford University Press.

- $\quad$ Pierre, J. \& peters, G. (2000). Governance Politics and State. Basingstoke: Macmillan.

- Ramírez, S. (2002). La compleja relación colombo-venezolana. Una coyuntura crítica a la luz de la historia. Análisis Político, 46,: 100-115.

- Ramírez, S. (2006). La integración y el desarrollo fronterizo. Cátedras de Integración Convenio Andrés Bello. Serie Integración Social y Fronteras. Bogotá: CAB.

- Ramírez, S. (2006). ¿De una aguda tensión a una asociación estratégica? En Socorro, R. \& Cárdenas, J. (Eds.) Colombia-Venezuela Retos de la integración. (p. 23 - 56). Bogotá: Unibiblos. 
- Rosenau, J. (1997). Cambio y Complejidad. Desafíos para la comprensión en el campo de las Relaciones Internacionales. Análisis Político, 32, 106-119.

- $\quad$ Rosenwurcel, G., Bezchinsky, G., Gianello, C. \& Thomas, Hernan. (2008). Innovación a escala Mercosur. Una vía para superar el estancamiento de la integración regional. Buenos Aires: Prometeo.

- Ruggie, J. (1993). Multilateralism Matters: the Theory and Praxis of an Institutional Form. New York: Columbia University Press.

- Scott, A. (1998). Flexible production systems and regional development: the rise of new industrial spaces in North American and Western Europe. International Journal of Urban and Regional Research, 12, 171185.

- Seguí, J. (1994). Análisis y estructuración de las redes en el espacio. En Gamir, A. \& Ruiz, M. (Cords.) Prácticas de Análisis Espacial. (p. 87-109). Barcelona: Oikos-Tau.

- Storper, M. (1995). The resurgence of regional economies, ten years later: the region as a nexus of untraded interdependences. European Urban and Regional Studies, 2, 191-221.

- Storper, M. (1997). The regional world: territorial development in a global economy. New York: Guilford.

- Stiglitz, J. (2002). El Malestar en la Globalización. Barcelona: Taurus.

- Strange, S. (2001). La retirada del Estado. La difusión del poder en la economía mundial. Barcelona: Oxfam.

- Soros, G. (1999). La crisis del capitalismo global: la sociedad abierta en peligro. Barcelona: Plaza \& Janés.

- Swyngedouw, E. 1997. Neither global nor local: Globalization and the politics of scale. En Cox, K. (Ed.) Spaces of Globalization Reasserting the Power of the Local. (p. 137-166). New York: Guilford.

- Tömmel, I. (2004). Las transformaciones de la gobernanza: La estrategia de la Comisión Europea a favor de la Europa de las regiones. En Morata, F. (Ed.) Gobernanza multinivel en la Unión Europea. (p. 87-120). Valencia: Tirant Lo Blanch.

- Wallerstein, I. (2004). Capitalismo histórico y movimientos antisistémicos: un análisis de sistemas-mundo. Madrid: Akal.

- Urry, J. (2003). Global Complexity. Cambridge: Polity.

- Vieira, E. (2008). La formación de espacios regionales en la integración de América Latina. Bogotá: Convenio Andrés Bello. 